\title{
LOCAL ADAPTIVE CONTRAST ENCHANCEMENT IN DIGITAL IMAGES
}

\author{
G. Apostolopoulos \\ Dept. of Electrical Engineering and \\ Computer Technology, \\ University of Patras \\ Kato Kastritsi \\ 2610996476, 26500 \\ gapost@george.wcl2.ee.upatras.gr
}

\author{
E. Dermatas \\ Dept. of Electrical Engineering and \\ Computer Technology, \\ University of Patras \\ Kato Kastritsi \\ 2610996476, 26500
}

dermatas@george.wcl2.ee.upatras.gr

\begin{abstract}
In this paper an algorithm for optical quality enhancement in low contrast images is proposed. The brightness dynamic region is increased using a non-linear transformation function, which is adapted from the local contrast. In a new approach, the local contrast is defined in every central pixel, using a four direction masking method and a statistical estimation of the local contrast value. Two adaptive thresholds locate the exact pixel positions where the proposed contrast adjustment algorithm is used to improve the image quality. In the evaluation process, several artificially distorted images are enhanced and the mean square error and peak noise ratio between the restored and the original images are estimated. The experimental results show both objective and subjective improvements in the image quality.
\end{abstract}

\section{Keywords}

Contrast, transformation function, image enhancement, pixel, digital image, dynamic region, brightnesses

\section{INTRODUCTION}

Contrast enhancement is one of the most important problems in image processing both digital and analog. In several cases the image acquired in severe conditions are too dark and blur and therefore difficult to recognize the different objects or scenery contained in the image. Therefore image enhancement algorithms are applied to improve the appearance of an image for human visual analysis or occasionally for subsequent machine analysis [1]. The image enhancement methodology dependents on the application context; criteria for enhancement are often subjective or too complex to be easily converted to useful tools. However, it is well known that a global image enhancement method for all classes of imagery is missing.

Permission to make digital or hard copies of all or part of this work for personal or classroom use is granted without fee provided that copies are not made or distributed for profit or commercial advantage and that copies bear this notice and the full citation on the first page. To copy otherwise, to republish, to post on servers or to redistribute to lists, requires prior specific permission and/or a fee. MOBIMEDIA 2007, August 27-29, Nafpaktos, Greece Copyright (C) 2007 ICST 978-963-06-2670-5 DOI 10.4108/ICST.MOBIMEDIA2007.1942
Classical techniques [2], [3] for digital image contrast enhancement are usually based on the analysis of the histogram, and known as point-operation global methods. In the global histogram equalization based methods, various constraints are added to the equalization procedure to achieve better performance. The global techniques completely fail when the original image is already occupying the full dynamic range, or when the contrast is varying in different parts of the image. Linear stretching of the brightness in monochrome images [4] are the standard methods to cover the available dynamic-range. Nonlinear functions like the exponential and logarithmic can also be used to preferentially stretch the contrast in bright and dark regions respectively. Adaptive histogram equalization techniques [5], [6] use statistical information in the neighborhood of each pixel to perform equalization. The major problem with adaptive histogram equalization methods is the over-enhancement effect, creating objects that were not visible in the original image. The enhanced image often does not look natural and is disturbing [7], [8], [9]. A multi-scale algorithm has been proposed in [10] to enhance the visibility of local features in an image, based on a gamma-like correction and multi-scale decomposition.

In this paper, we propose a contrast enhancement algorithm in both brightness and spatial domain. The basic idea of this approach is to enhance fully-automatically the optical quality of low contrast images. This is achieved by estimation of multiple central-pixel contrasts in four directions that differ 45 degrees. When the local contrast of an image is degraded, the contrast enhancement is achieved using a suitable transformation function. Using two thresholds, the brightness of central pixel changes with a specific condition: if the brightness exceeds a certain range (thresholds) its value remains unchanged otherwise the value of the pixel will be changed. The proposed algorithm performs partial transformation of the original image, increasing the contrast only in the low-quality regions.

The structure of the paper is as follows: in the next section the proposed method is described in detail. The experimental results applying on real images are presented and discussed in section 3 . Finally, the paper is concluded with directions for further algorithm improvement.

\section{ALGORITHM OVERVIEW}

Low-contrast images can result from, insufficient lighting, small dynamic region of optical sensor, natural phenomena (i.e. fog), incorrect setting of image acquisition systems (i.e. erroneous 
diaphragm settings). In digital imaging, the adjustment of contrast level increases the dynamic region of brightness. In the proposed algorithm an efficient transformation function increases adaptively the image contrast in predetermined regions. The flowchart of the proposed contrast enhancement algorithm is shown in figure 1.

Several definitions of the contrast have already been proposed [11]. Generally, the contrast definitions are close related to the mean variability of brightness in the image, giving a quantitative measure for the whole image contrast. Thus, for an (nxm) grayscale image of mean brightness $\mathrm{B}$, the contrast is given by the following relation [11]:

$$
C=\sqrt{\frac{1}{n \cdot m} \sum_{i=1}^{n} \sum_{j=1}^{m}[I(i, j)-B]^{2}},
$$

where $I(i, j)$ is the brightness value in pixel $(i, j)$.

In a great number of applications, a local definition of contrast is required, i.e. in a pixel based contrast only the brightness of the neighboring pixels should involved in the computations of (1).

Several definitions of contrast are in use in such different fields as psychology, optics and photography. In this paper, the contrast of a pixel is defined as the difference between its brightness and the corresponding average value of the neighbor pixels, normalized by the full dynamic range. A typical definition is as follows:

$$
C_{L}(i, j)=\left|\frac{I(i, j)-\bar{I}}{I_{\max }-I_{\min }}\right|
$$

This equation implies that the most direct method to enhance the image contrast is to stretch or expand the brightness from the corresponding mean value. It can be easily show that the local contrast is limited to $[-1,1]$, a similar definition space used to histogram equalization methods.

Extending the definition of local contrast, given in (2), the local contrast between two images or regions of an image A and B is defined by:

$$
C_{A B}=\left|\frac{\bar{I}_{A}-\bar{I}_{B}}{\bar{I}_{A}+\bar{I}_{B}}\right|,
$$

where $\bar{I}_{A}$ and $\bar{I}_{B}$ are the mean brightness of regions $\mathrm{A}$ and $\mathrm{B}$. If the region $\mathrm{A}$ is shrinking to a pixel an alternative definition of local contrast is reached:

$$
C(i, j)=\left|\frac{I(i, j)-I_{N}}{I(i, j)+I_{N}}\right|,
$$

where $I_{N}$ it is the mean brightness of a neighbor area for the pixel $(i, j)$.

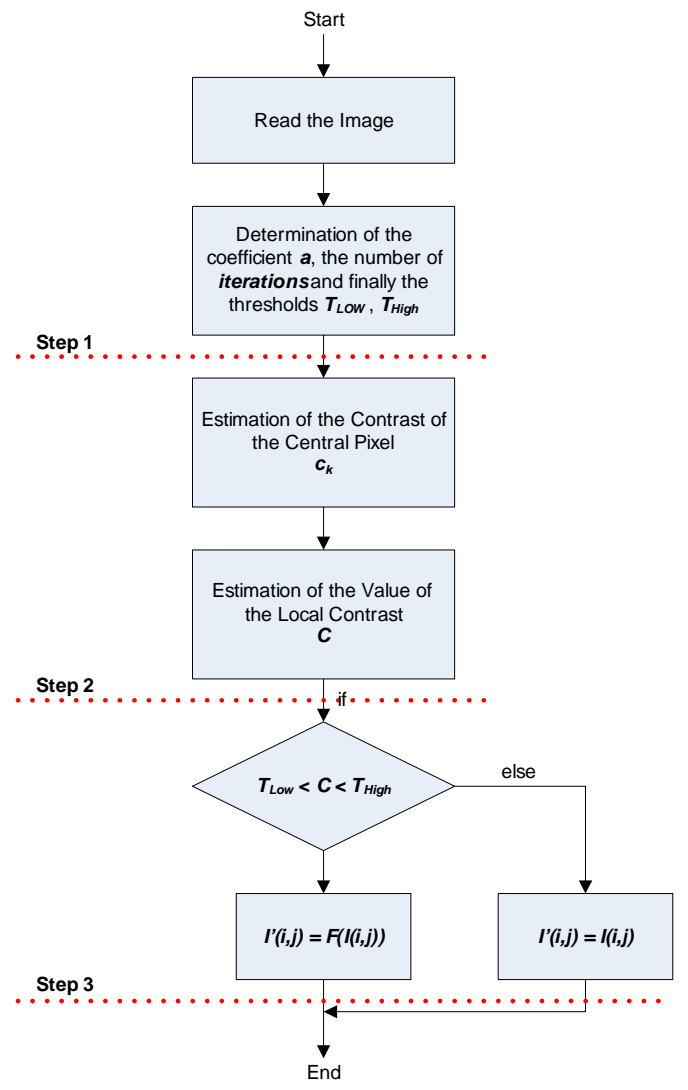

Figure 1. Flow-chart of the proposed algorithm

If the neighbor areas are defined in streight lines, a definition of the local directional contrast can be produced from the definition of (4) at different directions using a neighbor mask (with size $n \mathrm{x}$ $\mathrm{n}$ in this study). In this paper four straight departments are studied in which the pixels sub-sets concern differ 45 degrees each other, as shown in Fig. 2.

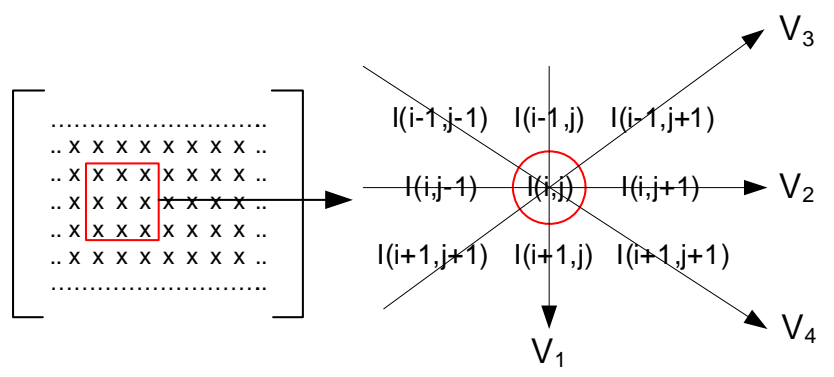

Where:

$$
\begin{aligned}
& V_{1}=\left[\begin{array}{lll}
I(i-1, j) & I(i, j) & I(i+1, j)
\end{array}\right] \\
& V_{2}=\left[\begin{array}{lll}
I(i, j-1) & I(i, j) & I(i, j+1)
\end{array}\right] \\
& V_{3}=\left[\begin{array}{lll}
I(i+1, j-1) & I(i, j) & I(i-1, j+1)
\end{array}\right] \\
& V_{4}=\left[\begin{array}{lll}
I(i-1, j-1) & I(i, j) & I(i+1, j+1)
\end{array}\right]
\end{aligned}
$$

Figure 2. Pixels involving in the estimation of local contrast 
The directional local contrast for the central pixel $(i, j)$ is:

$$
c_{k}=\frac{1}{n-1} \cdot \sum_{\substack{m=-1 \\ m \neq n / 2}}^{n} \frac{\left|V_{k}(n / 2)-V_{k}(m)\right|}{\left|V_{k}(n / 2)+V_{k}(m)\right|} \text {, where } k=1,2,3,4
$$

where $V_{k}(i, j)$ is the $\mathrm{k}^{\text {th }}$ directional vector of the pixel $(i, j)$ and $V_{k}(m)$ the $m^{\text {th }}$ element of the $V_{k}(i, j)$.

The four directional local contrasts are sorted in increased order, constructing the vector $L$ :

$$
L=\operatorname{sort}\left(c_{1}, c_{2}, c_{3}, c_{4}\right) .
$$

The proposed algorithm is applied in pixels where certain statistical conditions in vector $L$ are met. The statistical estimators involved [21] for contrast enhancement is the mean, median, maximum and minimum value [12].

Mean value: The mean value of the set $L$ elements.

Median value: The median value of the set $L$ elements is estimated as follows: If the set has an odd number of elements, then the value of the local contrast is equal to the median element, otherwise the value of the local contrast is equal to the mean value of the two neighboring median elements [13]. In (6), the number of elements is four, but if more directions are used in (5), the number of elements in $L$ will be changed.

Maximum and minimum value: The maximum and the minimum value of the set $L$ [14] are derived i.e. the first and the last element.

In the contrast enhancement algorithm one of the four statistical values is used to define the transformation mask: the selected statistical feature, derived from the directional local contrasts of the central pixel is compare to thresholds $T_{\text {Low }}$ and $T_{\text {High }}$ in [Imin,Imax]. If the statistical feature lies within two thresholds:

$$
T_{\text {Low }}<C<T_{\text {High }},
$$

C is the statistical feature of the central pixel, the pixel brightness is modified according to a predetermined transformation function otherwise, the pixel brightness remains the same. Thus, the contrast in homogeneous regions of the image remains the same, as well as in regions of high contrast

The total transformation scheme is given by:

$$
I^{\prime}(i, j)=\left\{\begin{array}{l}
F(I(i, j)), T_{\text {Low }}<C<T_{\text {High }} \\
I(i, j), \text { otherwise }
\end{array}\right.
$$

The function $F(I()$.$) , is called transformation brightnesses$ function, with $F$ definition space in [Imin,Imax].

Sigmoid function is a continuous nonlinear activation function. The name, sigmoid, obtained from the fact that the function is "S" shaped. The sigmoid function is continuous function, with limited output space ranging in the ordinary form in $[0,1]$. A more detail presentation of this function, refer to [15]. Linear transformation of the function, adapt the output space of $[0,1]$ to desired [Imin,Imax].
As shown in Fig. 4, the transformation function allocates three regions, one dark region, one saturation region and finally one linear-like region. The gradient of the straight line determines the dynamic region of the brightnesses. For values of brightnesses smaller than a threshold $g_{1}$, the sigmoid output belongs to the Imin neighbors and, similarly for values of brightnesses greater than $g_{2}$, the sigmoid output belongs to the Imax neighbors.

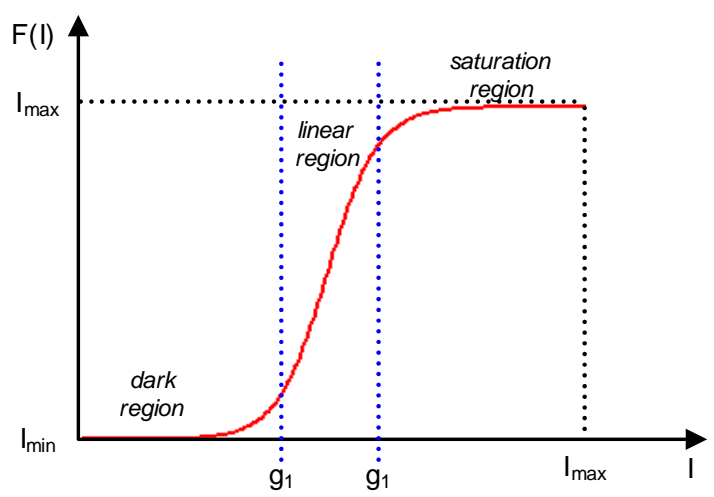

Figure 3. The sigmoid transformation function.

Consequently, the transformation function is determined as follows:

$$
F(I)=\frac{I_{\max }-I_{\min }}{1+e^{-\alpha \cdot\left(1-0.5 \cdot\left(I_{\max }-I_{\min }\right)\right)}}+I_{\min }
$$

The coefficient $\alpha$ controls the gradient of the linear-like region. In Fig. 4, several plots of the transformation function is given, for different values of $\alpha$.

The algorithm can be applied iterative until the quality of image become satisfactory, as proposed in [16].

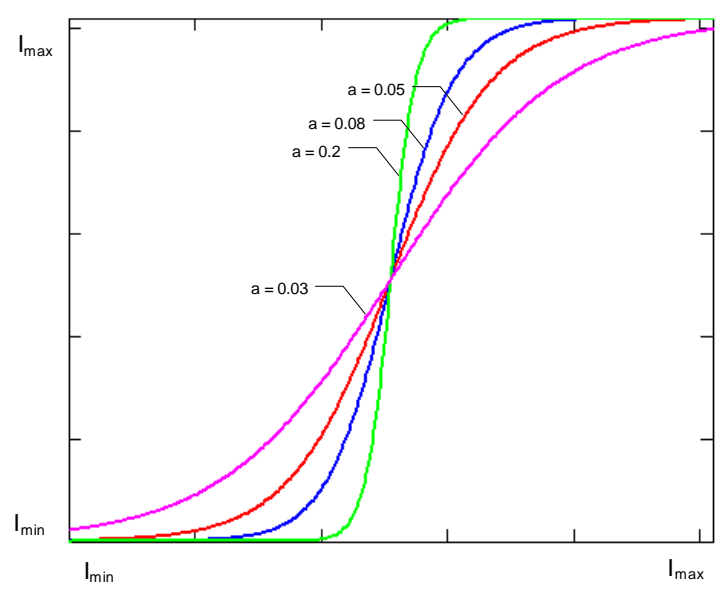

Figure 4. Plots of sigmoid function for various values of $\alpha$. Consequently, the relation (8) can be written as follows: 


$$
I^{(n+1)}=\left\{\begin{array}{l}
F\left(I^{(n)}(i, j)\right), T_{\text {Low }}<C^{n}<T_{\text {High }} \\
I^{(n)}(i, j), \text { otherwise }
\end{array}\right.
$$

where $n=1,2, \ldots .$. , is the iteration number.

In this paper, extensive experiments were carried out to locate an efficient method for robust estimation of algorithm parameters. The proposed technique consists of three steps. In the first step, the statistical feature is selected. In the second step, the coefficient $\alpha$ is determined followed by an estimation of the appropriate number of transformations and the definition of thresholds $T_{\text {low }}$ and $T_{\text {high }}$. In the last step the contrast enhancement algorithm is used to enhance the contrast in the automatically located areas.

\section{EXPERIMENTAL RESULTS}

The proposed algorithm is evaluated using several image data sets, downloaded from personal web-pages, or acquired using various digital cameras. The experimental results presented in this paper emanate from, (a) images in which their quality is degraded by adding fog-like noise, (b) images acquired by digital cameras, with presence of natural phenomena (e.g. fog) or low lighting. The proposed algorithm is used to improve the visual quality of these types of images acquired at 8 bits accuracy.

Many image processing methods are evaluated using a standard set of images, i.e. the image of Lena or the photographer. Examples include: image enhancement algorithms, image data compression and dithering algorithms. In the experimental evaluation, the popular mean squared error (MSE) or the peak signal noise ratio (PSNR, is a measure of the peak error), between the original and the enhanced image are estimated as an objective measure of visual quality. These measures are popular due to their analytical tractability [17]. Alternatively, the subjective human visual system is used to judge the image fidelity, taking into account that in several cases, better objective rates are in a disagreement to the rate given by human subjects.

The well known picture of Lena is presented in figure 5(a), while figure 5(b) shows the image after distortion with fog-like noise. The visual quality of the image has been strongly degraded.

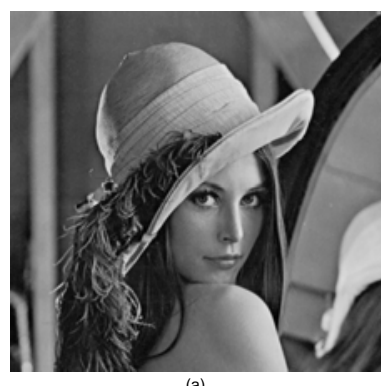

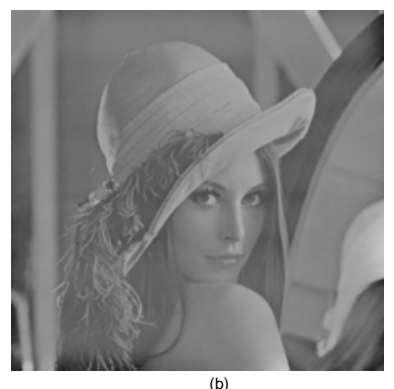

Figure 5. The Lena image and the distorted version

The contrast enhancement effect of the proposed algorithm is presented in Fig. 6, while in Table 1 the quantitative results for various values of the coefficient $\alpha$ are shown.
Table 1. MSE and PSNR between the enhanced and the original image of Lena

\begin{tabular}{c|llll}
\hline A & 0.03 & 0.04 & 0.046 & 0.06 \\
\hline MSE & 604.59 & 379.39 & 345.91 & 465.00 \\
PSNR (db) & 20.32 & 22.34 & 22.74 & 21.45
\end{tabular}

From the images of Fig. 6 it is obvious that the optical quality of the enhanced images have improved against the degraded picture of Fig. 5(b). For particular values of the coefficient $\alpha$ such as 0.03, 0.04, 0.046 and 0.06, the enhanced images are shown in Fig. 6(a), 6(b), 6(c) and 6(d) respectively.

In the subjective examination of the enhanced images a more or less psycho-optically criteria are involved based also in the knowledge of the original image. From the images of Fig. 6, the 6(c) contains the image which is the most similar to the original Lena image shown in Fig. 5(a). Moreover, in Table 1, the same image is rated with the minimum MSE of 345.91 among the four enhanced images with different coefficient $\alpha$. In this case, the best objective and subjective rate is achieved when the coefficient $\alpha$ has the value 0.046. In all images the threshold values $T_{\text {Low }}$ and $T_{\text {High }}$ was selected in the region of $[0,0.12]$ and only one iteration of the algorithm was applied.

A digital image of Patras lighthouse is presented in Fig. 7(a), while Fig. 7(b) shows the same image after artificial distortion with fog-like noise.

The experimental results of the image enhancement algorithm, is presented in Fig. 8, while in Table 2, the quantitative results are shown, for various values of the coefficient $\alpha$.

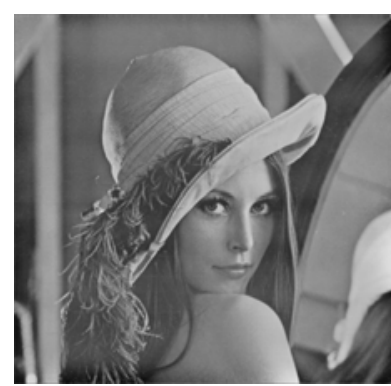

(a)

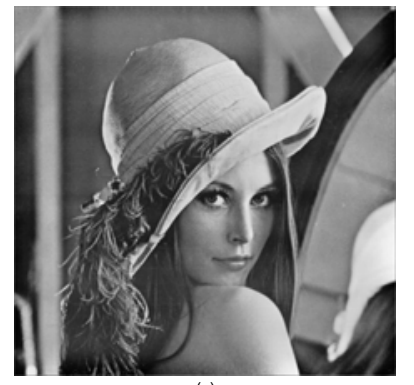

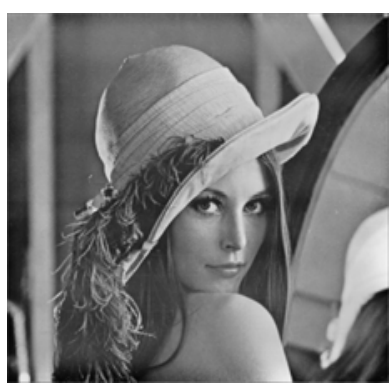

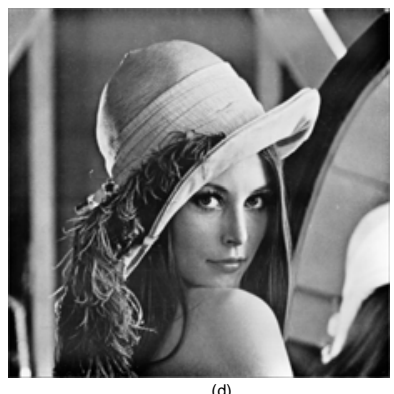

Figure 6. Lena images after contrast enhancement for different values of $\alpha$ 

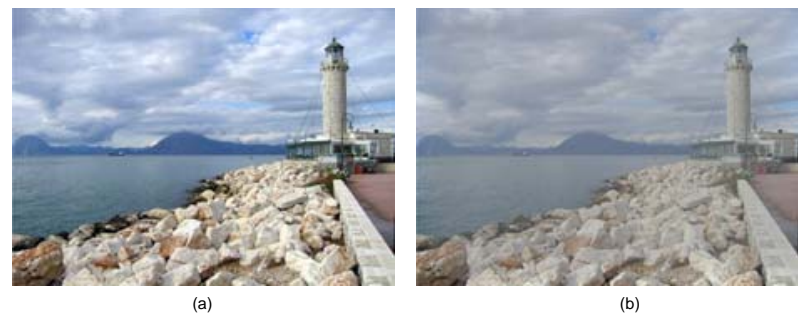

Figure 7. The Patras lighthouse and the distorted version

For particular values of coefficient $\alpha$, such as $0.02,0.031,0.04$ and 0.05 , the experimental results are shown in Fig. 8(a), 8(b), 8(c) and 8(d), respectively. The enhanced image, which is closest to the original Patras lighthouse, is shown in Fig. 8(c) or 8(d).

Table 2. MSE and PSNR between the enhanced and the original image of Patras lighthouse

\begin{tabular}{c|llll}
\hline $\mathbf{a}$ & 0.02 & 0.031 & 0.04 & 0.05 \\
\hline MSE & 407.77 & 121.43 & 271.91 & 633.42 \\
PSNR (db) & 22.03 & 27.29 & 23.79 & 20.11
\end{tabular}

From the MSE rates which given in Table 2, the image in Fig. 8 (b) is the closest to the original image. In this case the coefficient $\alpha$ has the value 0.031 . The PSNR criterion gives the best rate of 27.29 for the same image. The above images were resulted with thresholds values in region [0, 0.12], and one only iteration of the algorithm was applied.
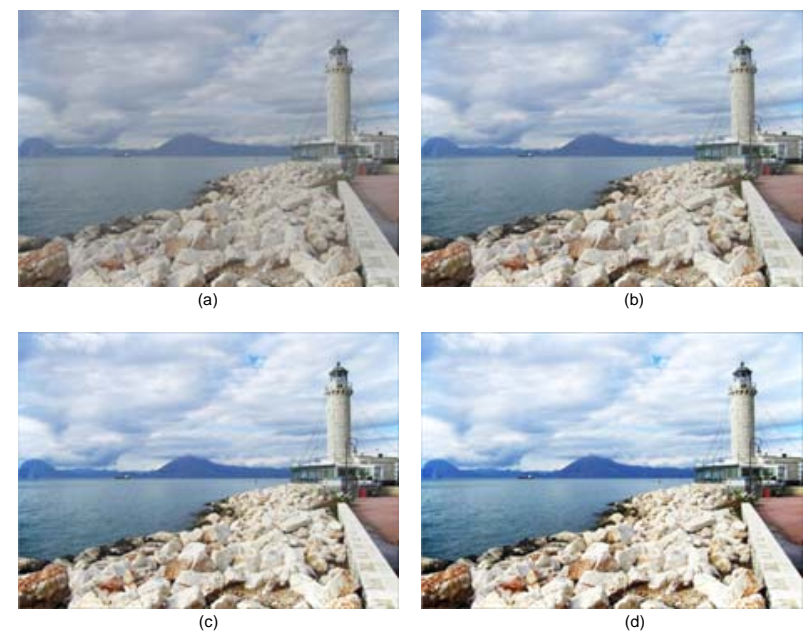

Figure 8. Images of Patras lighthouse after contrast enhancement for different values of $\alpha$

In the next experiment the proposed algorithm is evaluated in the presence of natural phenomenon (e.g. fog). In Fig. 9 an image of trees in fog is shown.

The image "suffer" from very low visibility, the brushes and the boles of the trees are distinguished with difficulty. The contrast enhancement results of the proposed algorithm, is presented in the image of Fig. 10.

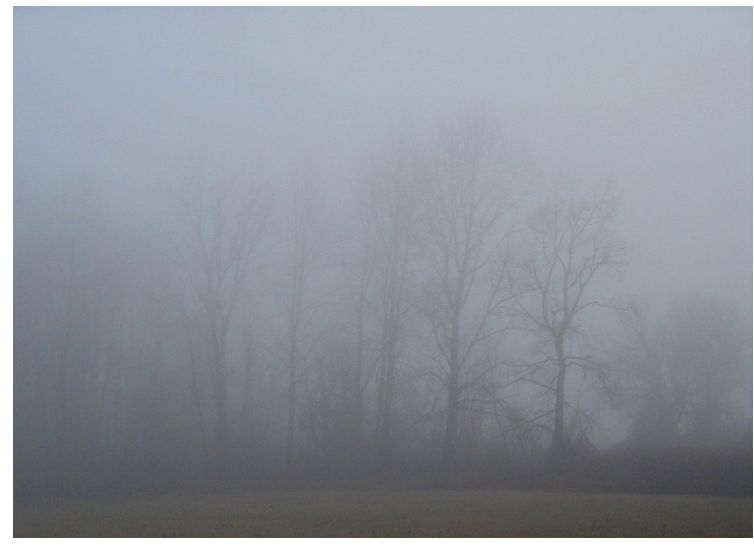

Figure 9. The original image of trees-in-fog.

In Table 3, the corresponding MSE and PSNR rates for various values of the coefficient $\alpha$ are shown estimated from the original (degraded) and the enhanced.

Table 3. MSE and PSNR of the trees-in-fog image

\begin{tabular}{c|llll}
\hline A & 0.03 & 0.035 & 0.04 & 0.05 \\
\hline MSE & 605.92 & 1000 & 14000 & 23000 \\
PSNR (db) & 20.31 & 18.07 & 16.52 & 14.45
\end{tabular}

The images in Fig. 10 are more luminous compared to the original image shown in Fig 9. The branches and the trunks of the trees are much more distinct than previously. Generally, the contrast enhancement algorithm improves the image perceptibility of the original degraded image. In this experiment we have a great disadvantage: the priori knowledge of the image without fog is missing, thus the best image judgment is clearly subjective and it is completely based on psycho-optically criteria. So, we consider that the image with better visual characteristics is created when the coefficient $\alpha$ takes the value 0.04 .

In Fig. 11, two contrast enhanced images of the trees-in-fog image are shown in which some of their regions are led to the saturation (255), while some others regions are led to the dark region (0). This effect is a result of high and low brightness in the original images (i.e. greater than 230, and lower than 30 ) in conjunction to high values of coefficient $\alpha$. In these two conditions are met, the brightness will be led either to the saturation region or to the dark region. The above images are created with thresholds values in region $[0,0.15]$ and one algorithm iteration.

The optimal results can be reached when the coefficient $\alpha$ and the number of algorithm iterations are carefully defined. The optimum definition of $\alpha$ and algorithm iterations remains an unsolved problem, and as the preliminary experiments shown is close related to the image content. 


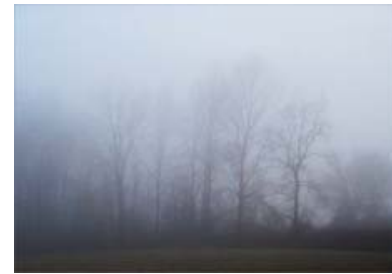

(a)

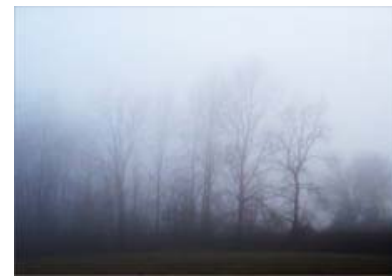

(c)

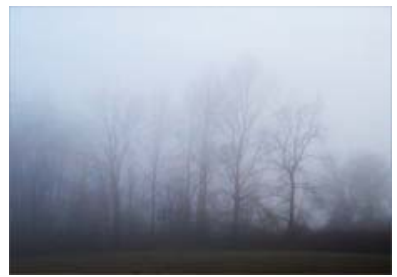

(b)

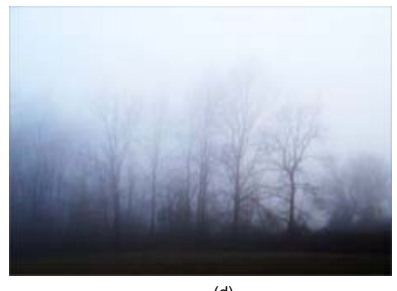

(d)
Figure 10. Images of trees-in-fog after contrast enhancement for different values of $\alpha$.

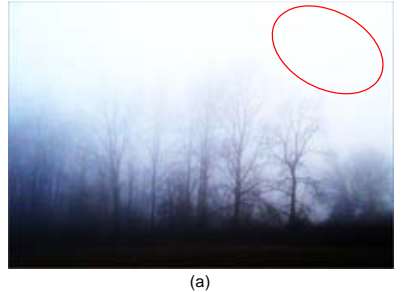

(a)

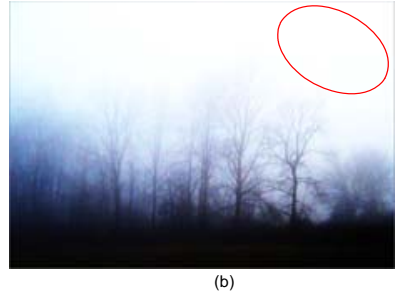

(b)
Figure 11. Trees-in-fog images, created with high values of coefficient $\alpha$.

\section{CONCLUSIONS}

In this paper, a robust contrast enhancement algorithm in low quality images has been presented, based in a local adaptive adjustment of the contrast. A mask, defined by two thresholds, is used to partially transform the image, enhancing the pixels suffering from low contrast. The experimental evaluation is applied to artificially and natural distorted grey-scale and color images show that the proposed algorithm is robust and able to recover images with very low contrast, so that their optical quality is significantly improved. There are several advantages of using this algorithm, including the ability to enhance the performance of any still image regardless of its construction, and to provide enhancements that are visually very difficult to achieve, beside its capabilities for enhancement of all types of photography, from snapshots to medical images. Further investigations have to be carried out to dynamically determine the appropriate coefficient $\alpha$ and the algorithm iterations number to achieve best objective and subjective rates.

\section{REFERENCES}

[1] J.R. Jenson, "Introductory digital image processing ”, Prentice Hall 2005

[2] Jain, A.k., 1989, "Fundamentals of digital image processing, Prentice-Hall, Eglewood Cliffs NJ
[3] Rosenfeld, A., and Kalk, A. C., 1982, "Digital Picture Processing”, Prentice Hall, Academic Press, San Diego

[4] J.DiCarlo and B. Wandell, "Rendering high dynamic range images," in Proc. SPIE Electronic Imaging, vol. 3965, (San Jose, CA), pp. 392-401, 2000.

[5] Pfizer S.M. et al, "Adaptive Histogram Equalization and its Variations", Computer Vision, Graphics and Image Processing, vol. 39, pp. 355-368, 1987.

[6] J.A. Stark and W.J. Fitzgerald, "An Alternative Algorithm for Adaptive Histogram Equalization”, Graphical Models and Image Processing,, vol.56, pp.180-185, 1996.

[7] J. A. Stark, "Adaptive image contrast enhancement using generalizations of histogram equalization," IEEE Transactions on Image Processing, vol. 9, no. 5, pp. 889-896, 2000.

[8] F.P.P. De Vries “Automatic, adaptive, brightness independent contrast enhancement”, Signal Processing vol. 21, pp. 169-182, 1990.

[9] J. L. Starck, F. Murtagh, E. Candes, and D. L. Donoho, "Gray and color image contrast enhancement by the curvelet transform," IEEE Trans. Im. Proc., vol. 12, pp. 706-717, 2003.

[10] N. Bonnier and E.P. Simoncelli, "Locally Adaptive Multiscale Contrast Optimization", Proc. 12th IEEE Intl. Conf. Image Processing Vol I, pp 949-952, Sep 2005.

[11] Seul M., O’Gorman L. and Sammon M., "Practical Algorithms for Image Analysis”, Cambridge University Press, 2001

[12] Hampel F., Ronchetti E., Rousseevw R., Stahel W. "Robust Statistics: An Approach Based on Influence Functions”, J. Wiley, 1986

[13] Tukey J.W., "Exploratory Data Analysis”, Addison Wesley, Reading MA, 1971

[14] Acre G.R and Mcloughlim M.P “ Theoretical analysis of the max/median filter”, IEEE Trans. on Acoustics, Speech and Signal Processing, Vol. 35, No. 1, 1987

[15] J. Hertz, R. Plamer, "Introduction to the neural computation", Addison Wesley, California, 1991

[16] Kramer H. and Bruchner J. "Iteration of a non-linear transformation for the enhancement of digital images", IEEE Trans. On Systems, Man and Cybernetics, Vol.7, pp.53-58, 1975

[17] Serra J. "Image Analysis and Mathematical Morphology", Academic Press, 1982

[18] Gongalez R. and Woods R., "Digital Image Processing", Addison - Wesley Publishing Co, 1993

[19] Beghdadi, A.,and Negrate, A. L., 1989, "Contrast enhancement technique based on local detection of edges", Computer Vision,Graphics and Image Processing, 46, 162-174.

[20] Korpi-Anttila, "Automatic color enhancement and scene change detection of digital video", Licentiate thesis, Helsinki University of Technology, Laboratory of Media Technology, 2003.

[21] D. L. Ruderman, “The statistics of natural images," Network: Computation in Neural Systems, vol. 5, pp. 517-548, 1996. 